
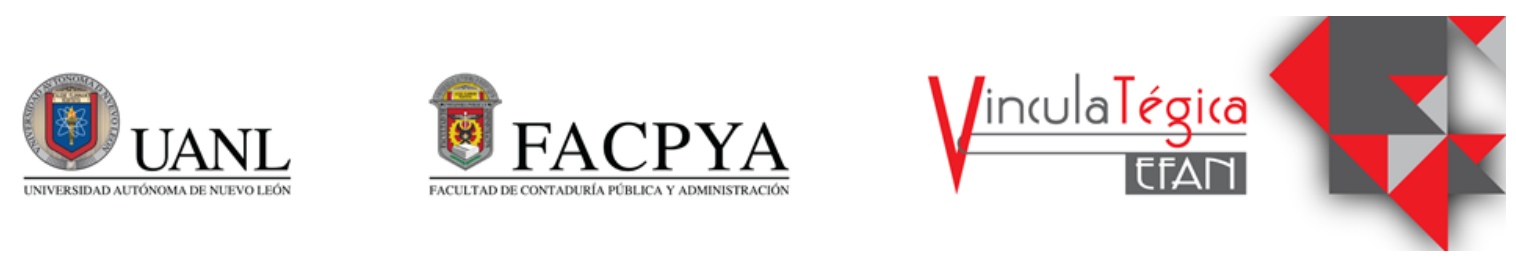

\title{
Diseño y validación del cuestionario sobre economía del aprendizaje de docentes universitarios
}

\author{
Susana Céspedes Gallegos ${ }^{1}$, Miguel Ángel Clara Zafra ${ }^{2}$ y Gilberto de León Cortés ${ }^{3}$ \\ ${ }^{1}$ Tecnológico Nacional de México Campus Coatzacoalcos, scespedesg@itesco.edu.mx, carretera Antigua a \\ Minatitlán KM 16.5, Reserva Territorial, C.P 96536 Coatzacoalcos, Ver., número de teléfono 9212118158 \\ ${ }^{2}$ Universidad Veracruzana Campus Xalapa, mclara@uv.mx, Lomas del Estadio s/n C.P 91000, número de \\ teléfono 2288421700 \\ ${ }^{3}$ Escuela Nacional de Protección Civil Campus Chiapas, gilberto.d64@yahoo.com, Chiapas, número de \\ teléfono961 6113599
}

\author{
Información del artículo revisado por pares \\ Fecha de aceptación: junio-2021 \\ Fecha de publicación en línea: diciembre-2021 \\ DOI: https://doi.org/10.29105/vtga7.1-150
}

\begin{abstract}
Resumen
La economía del conocimiento es la expresión acuñada para indicar que, en el estado actual del desarrollo, una parte sustancial de la producción se basa en el saber acumulado. La presente investigación muestra el resultado del diseño y construcción de un cuestionario validado sobre la percepción de factores que intervienen en el aprendizaje de docentes del nivel universitario, dicho cuestionario determinará si los factores facilitan u obstaculizan el aprendizaje de docentes. Cabe destacar que la investigación, siguió una metodología mixta, transversal y planteada desde el enfoque empírico-analítico. El método que se empleó fue el diseño de un cuestionario en diez faces: desde la revisión de la literatura hasta la consistencia interna del constructo, además de la revisión por expertos. Para la prueba piloto de donde se obtuvieron los datos, fue aplicada a 10 docentes de una Institución de Educación Superior (IES). Los resultados muestran un cuestionario con un Alfa de Cronbach 0.959, el cual denota la excelente consistencia interna, y se presenta con el propósito de que sea aplicada en IES para identificar áreas de oportunidad en su personal docente sobre el aprendizaje.
\end{abstract}

Palabras clave: Instrumento, Medición, Confiabilidad, Validez y Consistencia

\section{Clasificación Journal of Economic Literature} (JEL): 121, 123, 124

\begin{abstract}
The knowledge economy is the expression coined to indicate that, in the current state of development, a substantial part of production is based on accumulated knowledge. This research shows the result of the design and construction of a validated questionnaire on the perception of factors that intervene in the learning of teachers at the university level, said questionnaire will determine if the factors facilitate or hinder the learning of teachers. It should be noted that the research followed a mixed, cross-sectional methodology and raised from the empirical-analytical approach. The method used was the design of a ten-sided questionnaire: from the literature review to the internal consistency of the construct, in addition to the review by experts. For the pilot test from which the data were obtained, it was applied to 10 teachers from a Higher Education Institution (IES). The results show a questionnaire with a Cronbach's Alpha 0.959, which denotes the excellent internal consistency, and is presented with the purpose of being applied in IES to identify areas of opportunity in their personal teacher on learning.
\end{abstract}


Keywords: Instrument, Measurement, Reliability, Validity and Consistency

Journal of Economic Literature (JEL) Classification: $121,123,124$ 


\section{INTRODUCCIÓN}

Los cambios disruptivos del entorno han provocado que las Instituciones de Educación Superior (IES) se enfrenten a grandes retos que impactan en el sistema educativo, las técnicas aplicadas en el proceso de enseñanzaaprendizaje y el cumplimiento de planes de estudio se han tenido que adaptar a las nuevas condiciones del ambiente educativo. Por otro lado, la necesidad imperante de adquirir conocimientos de nuevas herramientas tecnológicas por parte de docentes ha sido crucial para la impartición de clases virtuales ante la contingencia sanitaria por COVID-19.

Ha sido una tarea compleja para los sistemas educativos adaptarse y enfrentar los retos que han derivado de la pandemia, sobretodo en la disposición de los docentes para aprender a desarrollar ambientes educativos virtuales. En este sentido, la presente investigación tiene como propósito diseñar y validar un instrumento de recopilación (cuestionario) para identificar los determinantes en el aprendizaje de los docentes universitarios, por otro lado, la encuesta permitirá a las IES emprender acciones y diseñar estrategias que brinde la posibilidad de establecer mejoras para la creación de ambientes de enseñanza, estudio y formación, que estimule a los docentes a la obtención de nuevos intelectos, habilidades y talentos en beneficio de la calidad educativa de la institución y de la comunidad estudiantil, en este mismo sentido, Céspedes, Ceja y de León (2020) consideran que una cultura ágil en las IES orientada hacia la innovación fomenta el trabajo colaborativo y permite alcanzar los objetivos propuestos.

\section{MARCO TEÓRICO}

\subsection{El estado de emergencia en las IES}

La actual emergencia sanitaria, social y económica afecta a todos los sectores productivos del país y por efecto a las IES, ya que no solo se enfrenta a la crisis financiera, sino a una situación que involucra personas, producción y bienestar, para lo cual instituciones como la Organizaciones de las
Naciones Unidas, ONU, el Fondo Monetario Internacional, FMI y el Banco Mundial, BM, favorecen a través de financiamiento.

$\mathrm{El}$ incremento en el uso del internet y de las tecnologías digitales han perpetuado la desigualdad entre las personas al no tener acceso tanto en el campo laboral como en el sistema educativo, para lo cual la Organización de la Naciones Unidas para la Educación, la Ciencia y la Cultura, UNESCO, (ONU, 2020) sostiene que se debe eludir el alarmismo, utilizar los sitios web y las redes sociales, utilizar telefonía móvil, capacitar al profesorado y garantizar la continuidad de los estudiantes con equidad. Existe una alta tasa de conectividad en el sistema, 1 de cada 2 hogares está conectado. En una IES, el 75\% de los estudiantes universitarios tienen acceso a la tecnología y el $25 \%$ se encuentra actualmente desconectado, ya que habitualmente acuden a sitios web cercanos a sus hogares o bien porque los estudiantes se encuentran en localidades donde la señal de internet no tiene acceso. Razón por la cual las IES deben planificar la docencia on-line para los semestres venideros.

Las IES deben transitar a ser instituciones educativas resilientes con perspectiva humanitaria. Actualmente las IES han apostado por el paradigma educativo de una calificación, para dar cumplimiento a un plan de estudio, ante este panorama qué se espera de las IES, enseñar "algo" que sirva para la escuela y no "algo" que sirva para la vida. Por tal motivo los docentes juegan un papel protagónico ya que su formación profesional y académica es clave para enfrentar este proceso de cambio complejo y multifacético, ya que también implica un cambio de mentalidad, actitud, adaptación y posibilitar el conocimiento en entornos flexibles. Es por ello la necesidad de analizar la percepción de los docentes del TECNM Campus Coatzacoalcos para identificar los determinantes del aprendizaje en un contexto educativo.

De acuerdo con Stiglitz y Greenwald (2019) una sociedad del aprendizaje se centra en el intelecto de raíz de las personas, la empresa y la comunidad en general y en cómo ese aprendizaje se modifica, se trasmite y se 
pone en práctica. Es decir, todo lo que el ser humano hace, la sociedad en general y las organizaciones requieren de un determinado aprendizaje. En ciertos contextos o circunstancias las instituciones pueden ser débiles para enfrentar retos que conlleven riesgos, pero el conocimiento de sus colaboradores es relevante para un desarrollo exitoso en una economía del aprendizaje o del conocimiento.

En una economía del conocimiento permite a las IES preocuparse por alcanzar metas y objetivos, promover la adaptación al cambio y compromiso social ante nuevos contextos o paradigmas del aprendizaje, obtener experiencias educativas exitosas en su personal docente, tomar en cuenta que en la medida en que los profesores aprendan brindarán la posibilidad de aumentar su rendimiento y productividad en la institución y el hecho de aprender nuevos conocimientos útiles debe provocar un impacto positivo en la institución pero sobre todo en la formación de jóvenes competitivos y cualificados ante un las demandas del mercado laboral.

La Organización de la Naciones para la educación, la Ciencia y la Cultura Unidas, UNESCO (2015) en su meta 4.3 acceso igualitario a la educación técnica/profesional y superior para el 2030, asegura debe de existir una condición igualitaria para tener acceso a la educación en todos los niveles educativos y en la meta 4.4 habilidades adecuadas para un trabajo decente, incrementar la cantidad de jóvenes y adultos para que tengan mayores competencias y habilidades para lograr una oportunidad de acceso al empleo, trabajo digno y al emprendimiento. En ese mismo sentido la UNESCO establece en el apartado 4C correspondiente a los maestros y educadores, incrementar significativamente la oferta de profesores capacitados, por medio del apoyo internacional para el desarrollo de las competencias docentes, en los países en vías de desarrollo, principalmente los subdesarrollados. Por lo que la preparación y actualización de los docentes es de vital importancia para la contribución académica y profesional de los jóvenes universitarios a nivel nacional e internacional.

Derivado de la actual situación de pandemia, hoy en día se requiere formar sociedades basadas en el aprendizaje para fomentar la investigación y el desarrollo de sus integrantes de tal forma que se de paso a la innovación y progreso entre los mismos y por ende en el país. Para Correo, Benjumea y Valencia (2019) un ingrediente principal en la educación es formar académicamente a los estudiantes con un pensamiento crítico y flexible orientado a un proyecto de vida, profesional y laboral, en este sentido, se necesita de profesores con vocación capaces de cumplir con su misión de manera determinante. La pandemia ha evidenciado a la mayoría de los IES con respecto a sus carencias y necesidades para enriquecer sus procesos de enseñanza-aprendizaje y la tecnología, que es en elemento clave para enriquecer dichos procesos, ya que permite acercar a los participantes involucrados (Rodríguez, 2019).

En el mismo sentido de la pandemia, Quintero (2020) señala que es posible que existan dos fenómenos importantes en las IES: deserción y abandono de los estudiantes con respecto a todos los niveles educativos debido a la carencia de la conectividad. Ante este reto según Suárez y Fernández (2018) las IES tienen que ser flexibles ya que en una economía de mercado existen elementos como la globalización y la competitividad que requieren del capital humano eficiente, por lo tanto, la educación es vital para el crecimiento y desarrollo económico y social de un país. Para lo que Barrios, Olivero y Figueroa (2020) sostienen que la economía de un país se encuentra en un contexto dinámico y cambiante en el que las tecnologías y los productos se innovan de manera rápida por lo que se requiere de gestores con capacidad creativa e innovadora para fomentar dichos cambios en los mercados.

\subsection{Hacia una economía del aprendizaje}

Es por ello, que las IES deben comprender, entender, reflexionar y adaptarse a los contextos emergentes y ser visionarias y predictores del futuro ya que en caso contrario sus metas serán irrelevantes y pocos competitivos en el mercado laboral (Álvarez, 
Arreguit, y Xavier, 2019). Las organizaciones requieren del talento de profesionales capaces de enfrentar retos y riesgos, pero sobre todo capaces de detectar amenazas y oportunidades que se puedan presentar desde diversos contextos económico, social, normativo, político, ambiental y cultural según los estudios de Roncancio (2020). Así como también que los futuros profesionistas puedan ser productivos y competitivos según Pulgarín, Ormaza y Erazo (2020) sostienen que el capital intelectual es uno de los recursos de los cuales disponen las organizaciones para incrementar su productividad laboral y está ligada al desempeño laboral de los colaboradores, aunado a la tecnología y los recursos de las organizaciones. Es importante señalar que el aprendizaje en las IES es un factor elemental para contribuir con la generación de conocimientos, saberes y competencias que permitan enriquecer el trabajo académico tanto de los profesores como de los estudiantes y que brinde la posibilidad de satisfacer los requerimientos de las empresas de la región y del país.

En caso contrario Lora, Castilla y Góez (2020), mencionan que los estudiantes pueden demostrar que no tienen los saberes, habilidades y talentes para determinados puestos debido a la debilidad académica en su formación profesional. En efecto, para Ramírez, Espíndola, Ruíz y Hugueth (2019) las IES tienen que considerar que la globalización, las Tecnologías de la Información, TICS, el mercado internacional, los contextos emergentes, las políticas gubernamentales, el intercambio intercultural y la pandemia actual requieren de conocimientos y aprendizajes para la solución de problemas que favorezcan la innovación y la apertura de nuevas formas de trabajo, de tal manera que se pueda dar origen a nuevos ambientes de aprendizaje.

Cabe señalar que existen otros conceptos relacionados con la economía del aprendizaje por lo cual es importante analizar sus diferencias:

La Economía de la educación tiene como principales precursores (Calderón, Ceccarini y Ríos, 2008):
- Schultz (1961), Denison (1962) y Becker (1962) proponen la Teoría de Capital Humano y el papel de la producción en la educación.

- Zvi Griliches (1969) considera que la inversión y la capacitación son elementos complementarios y se interrelacionan entre sí.

- Finis Welch (1970), Theodore Schultz (1975) y Jan Tinbergen (1975) analizan que el desarrollo de la tecnología incrementa la mano de obra calificada.

- Harry Braverman (1974) y Stephen Marglin (1974) observan que los cambios en las tecnologías afectan la mano de obra no calificada.

Una de las definiciones más aceptadas de dicho concepto es la de Cohn y Geske 1990 citado por Morduchowics (2004): "la economía de la educación es el estudio de cómo los hombres y la sociedad eligen, con o sin necesidad del uso de dinero, emplear recursos productivos escasos para producir diversos tipos de formación, el desarrollo de conocimientos, habilidades, carácter, etc. especialmente por medio de la educación formal- a través del tiempo para ser distribuida entre las distintas personas y grupos en la sociedad."

La economía del conocimiento cuenta con los principales antecedentes (BBVA, 2017):

- Foray (2204), Romer (1991), Grossman y Helpman (1991) y Salai Martín (2000) en el inicio de sus investigaciones aportan el término de conocimiento tecnológico.

- Stiglitz (2006) identifica el término de conocimiento acumulado

- Lall (1996) hace referencia a las capacidades tecnológicas en tres aspectos:

1. Incorporado: incluye el software, computadoras, máquinas $\mathrm{y}$ herramientas

2. Codificado: patentes, artículos científicos, manuales e instructivos.

3. Tácito: el capital humano en las organizaciones 
- Griliches (1990) acuña el término función de la producción del conocimiento, que incluye el conocimiento, el capital humano, investigación, desarrollo e infraestructura.

- Schmokler considera que producir conocimiento en las organizaciones tiene un costo relativamente alto.

La economía del conocimiento es la expresión acuñada para indicar que, en el estado actual del desarrollo, una parte sustancial de la producción se basa en el saber acumulado: capital humano y el capital tecnológico. Se considera que el conocimiento es la pieza clave para impulsar el avance tecnológico, la productividad, la investigación, la ciencia, el desarrollo e innovación de un país, a través del capital humano, lo cual potencializa el desarrollo de los países (Clara y Vega, 2020). En este sentido la economía del conocimiento descansa en cuatro pilares:

- Una fuerza de trabajo educada y calificada,

- Una infraestructura de información sólida y actualizada,

- Un sistema de innovación e

- Incentivos.

Después de analizar lo antes expuesto, la economía del aprendizaje es un tema relativamente nuevo en su campo de estudio y en este sentido de acuerdo con Stiglitz y Greenwald (2019) se define como el conocimiento arraigado en los individuos y cómo ese conocimiento se trasmite y se lleva a la práctica, para ello los individuos deben tener una especie de motivación hacia el aprendizaje. El conocimiento se origina y se desarrolla con el trabajo de los colaboradores al interior de las organizaciones y se difunde a otros elementos de la organización. El conocimiento es el elemento clave para estimular a sus integrantes para el surgimiento de la creatividad e innovación. De acuerdo con Céspedes, Pacheco y Vázquez (2020) establecen que hoy en día las organizaciones necesitan de líderes transformadores capaces de inducir un cambio en los colaboradores para ser creativos e innovadores precisamente a través del conocimiento. Los individuos y las organizaciones requieren del aprendizaje para encontrar la mejor forma de hacer las cosas utilizando menos recursos y tiempo. De acuerdo con Nordhaus (1962) considerado como uno de los principales pioneros de la economía del aprendizaje involucra el término de "aprender haciendo", en el sentido de asignar recursos para fomentar la investigación y el desarrollo en las organizaciones. En ese mismo sentido Nordhaus (1969), sostiene que se necesitan verdaderos cambios transformadores en las organizaciones acompañados de inversión para apoyar la generación de ideas. Ambos autores consideran que el aprendizaje es indispensable para dar paso a la investigación y desarrollo en las organizaciones.

Una teoría como antecedente del aprendizaje es la ventaja comparativa redefinida de Heckscher y Ohlin, la cual argumentan que el aprendizaje necesita de una dotación de factores: el estado del conocimiento y las capacidades del aprendizaje. Consideran que el aprendizaje es el resultado de aprender de la experiencia, de aprender de otros y de la oportunidad de descubrir qué ideas, estrategias y prácticas utilizan otras empresas para aprender de ellas. Para efectos del presente estudio el término de aprendizaje está enfocado en el crecimiento y desarrollo sostenidos. Stiglitz y Greenwald (2019) proponen una economía del aprendizaje basada en los siguientes determinantes:

- Capacidades de aprendizaje: se refiere a la capacidad de aprender y que el aprendizaje económico ocurre en el trabajo, un elemento primordial en el aprendizaje es la educación.

- Acceso al conocimiento: Todo el aprendizaje se construye sobre los cimientos del conocimiento preexistente. Dentro de las organizaciones debe de existir una apertura que asegure el acceso al conocimiento.

- Catalizadores: El aprendizaje necesita que las personas y las empresas cuenten con capacidades de 
aprendizaje, pero deben ser motivados para lograrlo.

- Contactos: Es importante aprender de otras personas, estas relaciones aportan la materia prima del conocimiento que conforma la estructura del aprendizaje como un detonador que da origen a la innovación.

- Marcos cognitivos: Las personas y las organizaciones tienen que adoptar una ideología que de paso al aprendizaje. Esto incluye la credibilidad de que el cambio es persistente y estable.

- Contexto: El aprendizaje se genera dentro de un contexto, es decir, dentro de las organizaciones y para ello se necesita de una cultura del aprendizaje

Después de analizar los términos de economía del aprendizaje, de la educación y del conocimiento, se analiza el proceso de aprendizaje en las organizaciones:

- Aprendizaje: investigación formal, trabajo individual de desarrollo y experiencia propia.

- Educación: Capacitación del capital humano, formación intelectual.

- Conocimiento: Adquisición de nuevos "saberes" y "competencias".

- Transferencia del conocimiento e información: compartir con profesionales, estudiantes y expertos conocimientos sobre un tema en común por iniciativa propia, mediante el trabajo en equipo y la comunicación.

- Sociedad del conocimiento: establecer vinculación entre educación y desarrollo económico.

\section{Objetivo de la investigación}

Diseño y construcción de un instrumento de factores determinantes en el aprendizaje de los docentes en una IES.

\section{MÉTODO}

La presente investigación se plantea desde el enfoque empírico-analítico de tipo transversal (Ortiz, 2015), con un análisis de datos cuantitativos (Hernández, Fernández y Baptista, 2014) para la validación de una escala para determinar el grado de aprendizaje de docentes universitarios.

\subsection{Procedimiento de la escala}

Para el procedimiento de construcción y validación de la escala para medir la economía del aprendizaje en los docentes universitarios. Para ello, se siguió el procedimiento metodológico de Supo (2013), el cual consistió en:

1. La revisión de la literatura,

2. Se exploró el concepto,

3. Elección de variables,

4. Enlista de los ítems,

5. Selección de los jueces;

La valoración de expertos consistió en elegir a 10 investigadores y derivado de la disponibilidad 6 procedieron a realizar la valoración como especialistas en el tema. Posterior a la revisión, se atendieron los comentarios y sugerencias de los investigadores se construyó la escala.

6. Prueba piloto;

Se llevó a cabo una prueba piloto, la cual consistió en aplicar la escala a una muestra objetivo, por ello, se recurrió a un muestreo no probabilístico a conveniencia (Otzen y Manterola, 2017), y se obtuvo la participación de 10 docentes. El cuestionario con escala tipo Likert se aplicó a través de la aplicación de Google drive, en el período comprendido de julio-septiembre de 2020.

7. Evaluar la consistencia

8. Reducción de ítems

9. Reducción de dimensiones

10. Escala validada

En este sentido, los puntos antes descriptos marcan la pauta del procedimiento para la validación del cuestionario. A continuación, en la Tabla 1, se presenta el análisis de correlación y Alfa de Cronbach con el propósito de identificar la consistencia interna de la escala (Celina y Campo, 2005), 
dicho cálculo es de suma importancia para posteriormente realizar la validez interna, de constructo, criterio, estabilidad y rendimiento de la escala para la cual fue diseñada. Todo el procedimiento estadístico se ocupó el software SPSS V.25.

Tabla 1 Correlación de ítems y Alfa de Cronbach

Estadísticos total-elemento

\begin{tabular}{|c|c|c|c|c|}
\hline & $\begin{array}{l}\text { Media de la escala si se } \\
\text { elimina el elemento }\end{array}$ & $\begin{array}{l}\text { Varianza de la escala si } \\
\text { se elimina el elemento }\end{array}$ & $\begin{array}{l}\text { Correlación elemento- } \\
\text { total corregida }\end{array}$ & $\begin{array}{l}\text { Alfa de Cronbach si se } \\
\text { elimina el elemento }\end{array}$ \\
\hline $\mathrm{P} 1$ & 62.500 & 451.611 & .563 & .956 \\
\hline P2 & 62.000 & 434.000 & .810 & .953 \\
\hline P3 & 62.000 & 438.222 & .890 & .953 \\
\hline P4 & 62.100 & 429.878 & .764 & .954 \\
\hline $\mathrm{P} 5$ & 62.800 & 436.178 & .898 & .953 \\
\hline P6 & 62.000 & 425.556 & .757 & .954 \\
\hline P7 & 62.700 & 452.456 & .526 & .956 \\
\hline $\mathrm{P} 8$ & 62.300 & 436.011 & .930 & .953 \\
\hline $\mathrm{P9}$ & 63.000 & 442.444 & .780 & .954 \\
\hline P10 & 62.800 & 446.178 & .744 & .955 \\
\hline P11 & 62.600 & 445.600 & .595 & .955 \\
\hline $\mathrm{P} 12$ & 61.300 & 440.011 & .619 & .955 \\
\hline $\mathrm{P} 13$ & 62.600 & 454.267 & .520 & .956 \\
\hline P14 & 62.600 & 438.933 & .744 & .954 \\
\hline $\mathrm{P} 15$ & 62.900 & 438.322 & .733 & .954 \\
\hline $\mathrm{P} 16$ & 63.000 & 439.333 & .766 & .954 \\
\hline P17 & 61.800 & 439.733 & .613 & .955 \\
\hline P18 & 60.600 & 439.600 & .539 & .956 \\
\hline P19 & 62.400 & 420.489 & .802 & .953 \\
\hline P20 & 62.500 & 452.056 & .322 & .959 \\
\hline $\mathrm{P} 21$ & 61.200 & 437.067 & .589 & .956 \\
\hline $\mathrm{P} 22$ & 62.200 & 431.511 & .784 & .954 \\
\hline $\mathrm{P} 23$ & 61.300 & 451.344 & .625 & .955 \\
\hline P24 & 62.700 & 425.567 & .759 & .954 \\
\hline $\mathrm{P} 25$ & 62.400 & 431.378 & .798 & .953 \\
\hline P26 & 61.200 & 447.289 & .350 & .959 \\
\hline
\end{tabular}

Fuente: Elaboración propia (2021)

De acuerdo con Cohen y Manion (2002), en la columna de correlación-elemento total corregida, los ítems no deben ser menor a 0.350 , por lo cual, el ítem P20 debe ser eliminado para no afectar la confiabilidad del instrumento.

\section{RESULTADOS}

Validez interna

Para determinar la confiabilidad del 
instrumento se utilizó el coeficiente de Alfa de Cronbach, de acuerdo con Tavakol y Denniks, 2011 citado por Hernández, Fernández y Baptista (2014) consideran que el coeficiente debe ser entre 0.70 y 0.90 como válido.

Cabe recordar que la validación interna consistió en la validez de la escala por medio de 6 expertos investigadores nacionales e internacionales, quienes estimaron y analizaron la claridad, transparencia y coherencia de cada uno de los ítems que conforman el instrumento. Por otro lado, en la validación interna en la Tabla 2 , se presenta el coeficiente de Alfa de Cronbach, el cual denota una consistencia excelente.

\section{Tabla 2 Alfa de Cronbach}

Estadísticos de fiabilidad

\begin{tabular}{r|r}
\hline Alfa de Cronbach & N de elementos \\
\hline .956 & 26
\end{tabular}

Elaboración propia (2021)

\section{Validez de constructo}

Consiste en la simplificación de los ítems que constituyen el instrumento, en la Tabla 3, se analiza la correlación y el Alfa de Cronbach de cada uno de los ítems que conforman el instrumento. Se elimina el ítem P20 por no cumplir con los criterios correspondientes y se actualiza la tabla con el elemento eliminado.

Tabla 3 Correlación y Alfa de Cronbach con la eliminación del ítem Estadísticos total-elemento

\begin{tabular}{|c|c|c|c|c|}
\hline & $\begin{array}{l}\text { Media de la escala si se } \\
\text { elimina el elemento }\end{array}$ & $\begin{array}{l}\text { Varianza de la escala si se } \\
\text { elimina el elemento }\end{array}$ & $\begin{array}{l}\text { Correlación elemento- } \\
\text { total corregida }\end{array}$ & $\begin{array}{l}\text { Alfa de Cronbach si se } \\
\text { elimina el elemento }\end{array}$ \\
\hline $\mathrm{P} 1$ & 60.300 & 430.456 & .544 & .958 \\
\hline $\mathrm{P} 2$ & 59.800 & 412.844 & .804 & .956 \\
\hline $\mathrm{P} 3$ & 59.800 & 415.956 & .910 & .956 \\
\hline $\mathrm{P} 4$ & 59.900 & 407.878 & .777 & .956 \\
\hline P5 & 60.600 & 415.156 & .886 & .956 \\
\hline $\mathrm{P} 6$ & 59.800 & 403.511 & .771 & .956 \\
\hline P7 & 60.500 & 431.833 & .493 & .959 \\
\hline P8 & 60.100 & 414.322 & .936 & .955 \\
\hline P9 & 60.800 & 420.400 & .791 & .957 \\
\hline $\mathrm{P} 10$ & 60.600 & 424.044 & .756 & .957 \\
\hline $\mathrm{P} 11$ & 60.400 & 425.156 & .566 & .958 \\
\hline P12 & 59.100 & 417.433 & .639 & .958 \\
\hline P13 & 60.400 & 433.156 & .498 & .959 \\
\hline P14 & 60.400 & 417.600 & .739 & .957 \\
\hline $\mathrm{P} 15$ & 60.700 & 417.567 & .716 & .957 \\
\hline P16 & 60.800 & 418.844 & .740 & .957 \\
\hline $\mathrm{P} 17$ & 59.600 & 418.711 & .602 & .958 \\
\hline P18 & 58.400 & 417.822 & .542 & .959 \\
\hline P19 & 60.200 & 399.289 & .803 & .956 \\
\hline $\mathrm{P} 21$ & 59.000 & 416.222 & .577 & .959 \\
\hline $\mathrm{P} 22$ & 60.000 & 408.667 & .814 & .956 \\
\hline $\mathrm{P} 23$ & 59.100 & 428.767 & .647 & .958 \\
\hline P24 & 60.500 & 404.722 & .752 & .957 \\
\hline
\end{tabular}




\begin{tabular}{c|c|c|c|c} 
P25 & 60.200 & 409.956 & .800 & .956 \\
\hline P26 & 59.000 & 422.889 & .390 & .962
\end{tabular}

Fuente: Elaboración propia (2021)

En ese sentido, se puede observar que la eliminación del ítem P20, denota el aumento de la correlación-elemento del ítem P26, lo cual incrementa considerable su consistencia interna del cuestionario.

\section{Validez de criterio}

En este punto se confirman los resultados obtenidos. Se elimina el ítem P20 y se analizan los siguientes resultados en la tabla de frecuencia de los ítems. En la Tabla 4, se observan los resultados obtenidos de la asimetría y curtosis del ítem P20.

Tabla 4

Asimetría y curtosís del ítem P20

\begin{tabular}{l|l|r}
\multicolumn{3}{c}{ Estadísticos } \\
\hline \multicolumn{2}{l}{ P20 } \\
\hline \multirow{2}{*}{$\mathrm{N}$} & Válidos & 10 \\
\cline { 2 - 3 } & Perdidos & 0 \\
\hline Asimetría & .871 \\
\hline Error típ. de asimetría & .687 \\
\hline Curtosis & -.513 \\
\hline Error típ. de curtosis & 1.334
\end{tabular}

Fuente: Elaboración propia (2021)

La asimetría es la diferencia entre las partes o el punto en que la forma de la distribución de los datos no es simétrica y la curtosis indica la cantidad de datos que están cerca de la media.
En la Tabla 5, se observa la frecuencia obtenida en el ítem P20.

Tabla 5 Frecuencia del ítem P20

$\mathrm{P} 20$

\begin{tabular}{l|l|r|r|r|r}
\hline \multicolumn{2}{l|}{} & $\begin{array}{c}\text { Frecue } \\
\text { ncia }\end{array}$ & $\begin{array}{c}\text { Porcen } \\
\text { taje }\end{array}$ & $\begin{array}{c}\text { Porcentaj } \\
\text { e válido }\end{array}$ & $\begin{array}{c}\text { Porcentaj } \\
\text { e } \\
\text { acumula } \\
\text { do }\end{array}$ \\
\hline \multirow{4}{*}{$\begin{array}{l}\text { Váli } \\
\text { dos }\end{array}$} & 1.0 & 5 & 50.0 & 50.0 & 50.0 \\
\cline { 2 - 7 } & 2.0 & 1 & 10.0 & 10.0 & 60.0 \\
\cline { 2 - 6 } & 3.0 & 2 & 20.0 & 20.0 & 80.0 \\
\cline { 2 - 6 } & 4.0 & 1 & 10.0 & 10.0 & 90.0 \\
\cline { 2 - 6 } & 5.0 & 1 & 10.0 & 10.0 & 100.0 \\
\cline { 2 - 6 } & $\begin{array}{l}\text { Tot } \\
\text { al }\end{array}$ & 10 & 100.0 & 100.0 & \\
& & & &
\end{tabular}

Fuente: Elaboración propia (2021)

Derivado de las condiciones anteriores de la eliminación del ítem, se calcula de nueva cuenta el Alfa de Cronbach del instrumento de medición. En la Tabla 6, se observa de nueva cuenta el Alfa de Cronbach. 
Tabla 6 Alfa de Cronbach del ítem eliminado

Estadísticos de fiabilidad

\begin{tabular}{r|r}
\hline $\begin{array}{c}\text { Alfa de } \\
\text { Cronbach }\end{array}$ & \multicolumn{1}{|c}{$\begin{array}{c}\text { N de } \\
\text { elementos }\end{array}$} \\
\hline .959 & 25
\end{tabular}

Fuente: Elaboración propia (2021)

Como se puede observar el Alfa de Cronbach

aprecia el Alfa de Cronbach por dimensión del se ha incrementado de 0.956 a 0.959 con 25

ítems. A continuación, en la Tabla 7, se

Tabla 7 Alfa de Cronbach por dimensión del instrumento

\begin{tabular}{l|c|c}
\multicolumn{1}{c|}{ Dimensión } & Ítems & Alfa de Cronbach \\
\hline D1 Capacidad del aprendizaje & $1-4$ & 0.911 \\
\hline D2 Acceso al conocimiento & $5-9$ & 0.879 \\
\hline $\begin{array}{l}\text { D3 Catalizador para el } \\
\text { aprendizaje }\end{array}$ & $10-14$ & 0.887 \\
\hline D4 Contactos & $15-17$ & 0.781 \\
\hline D5 Marcos cognitivos & $18-19$ y 21 & 0.774 \\
\hline D6 Contexto & $22-26$ & 0.818
\end{tabular}

Fuente: Elaboración propia (2021)

\section{Estabilidad}

Con la finalidad de realizar el ejercicio de la investigación, se considera obtener en igualdad de condiciones, los resultados a través del tiempo y por el administrador del cuestionario en otras IES.

\section{Rendimiento}

La investigación y la propuesta de diseño del cuestionario se producen con fines de diagnóstico, para dar paso al análisis y el enriquecimiento del conocimiento en las IES.

\subsection{Cuestionario validado}

A continuación, en la Tabla 8, se presenta la escala del instrumento final, validada por expertos y estadísticamente.

\section{Tabla 7 Alfa de Cronbach por dimensión del instrumento}

\begin{tabular}{l|l} 
Ítems & \multicolumn{1}{|c}{ Pregunta } \\
\cline { 2 - 2 } & El docente percibe que... \\
\hline P1 & $\begin{array}{l}\text { la institución educativa apoya los esfuerzos del docente, a través de becas, programas de estudio y carga horaria, } \\
\text { para obtener un grado académico para enriquecer su labor docente mediante una especialización, maestría o } \\
\text { doctorado. }\end{array}$ \\
\hline P2 & $\begin{array}{l}\text { la institución proporciona cursos de actualización docente y profesional de acuerdo con sus necesidades de los } \\
\text { profesores. }\end{array}$ \\
\hline P3 & $\begin{array}{l}\text { la institución difunde de manera constante y con antelación, las diversas convocatorias para participar con la } \\
\text { comunidad estudiantil en las diversas áreas del conocimiento. }\end{array}$ \\
\hline P4 & $\begin{array}{l}\text { la dirección general y las jefaturas se preocupan por promover la educación continua hacia el mejoramiento de } \\
\text { la calidad docente. }\end{array}$ \\
\hline P5 & $\begin{array}{l}\text { la institución posee los medios tecnológicos para vincular su trabajo docente, como centros de cómputo } \\
\text { equipados, biblioteca digital, redes de investigación, estancias a nivel nacional e internacional, centros de } \\
\text { investigación y la vinculación con el sector empresarial para realizar trabajos conjuntos. }\end{array}$ \\
\hline P6 & se puede acceder fácilmente a las plataformas digitales con que cuenta la institución educativa \\
\hline P7 & la institución educativa adquiere libros y revistas especializadas relacionadas con su labor docente \\
\hline P8 & se cuenta con las herramientas digitales para el desempeño de su labor docente.
\end{tabular}




\begin{tabular}{l|l} 
P9 & se dispone de libros digitales y repositorios en la institución para fortalecer su labor docente. \\
\hline P10 & la institución dispone con incentivos económicos para la realización de proyectos productivos y de investigación. \\
\hline P11 & su labor docente es reconocida por la institución. \\
\hline P12 & el trabajo de los compañeros lo motiva a mejorar su labor docente. \\
\hline P13 & la institución otorga el crédito correspondiente al docente en la realización de algún logro académico. \\
\hline P14 & los proyectos son vinculados al sector empresarial o algún centro de investigación para la mejora de este. \\
\hline P15 & la institución realiza una vinculación a nivel nacional e internacional para enriquecer la labor educativa. \\
\hline P16 & las redes sociales de la institución educativa tienen un impacto a nivel nacional e internacional. \\
\hline P17 & $\begin{array}{l}\text { los compañeros de trabajo poseen contacto con instituciones a nivel nacional e internacional para enriquecer el } \\
\text { trabajo docente y colaborativo. }\end{array}$ \\
\hline P18 & $\begin{array}{l}\text { ante la situación de la pandemia del COVID-19 es importante generar cambios en la labor docente, } \\
\text { administrativa e institucional. }\end{array}$ \\
\hline P19 & la institución fomenta y promueve estrategias de cambio y motiva al personal para promoverlo. \\
\hline P20 & existe una adaptación y la de sus compañeros ante la nueva normalidad de trabajar en línea. \\
\hline P21 & existe un ambiente de aprendizaje en la institución. \\
\hline P22 & la labor docente de sus compañeros fomenta el aprendizaje en la institución. \\
\hline P23 & el jefe de carrera fomenta a través del ejemplo, cómo deben desarrollar de la mejor forma su labor docente. \\
\hline P24 & existe preocupación por parte de la institución para la creación de ambientes de aprendizaje. \\
\hline P25 & existe la necesidad de tomar la iniciativa de adquirir nuevos conocimientos asociados con su labor docente. \\
\hline Nota
\end{tabular}

Nota: las dimensiones se distribuyen: D1(1-4), D2(5-9), D3(10-14), D4(15-17), D5(18-20), y D6(21-25).

Fuente: Elaboración propia (2021)

Cabe destacar que se propone una escala tipo Likert para medir cada ítem, donde 1 es siempre y 5 es nunca. Además, se recomienda cuestionar otras preguntas de identificación edad, sexo, tipo de contratación, etc.

Los determinantes del aprendizaje tienen como finalidad la creación de una economía y una sociedad del aprendizaje, dicha taxonomía proporciona los ingredientes fundamentales para el diseño de una arquitectura del aprendizaje, es decir, capacidades para aprender, en el cual, la educación es el principal elemento. El aprendizaje económicamente relevante ocurre en el trabajo, no en la educación formal o académica, pero ambas son complementarias para mejorar la productividad en dichas instancias. El elemento primordial en la economía del aprendizaje es la cultura institucional en las IES.

Este estudio brinda la posibilidad de analizar los determinantes del aprendizaje de los docentes de una IES, pero es importante reconocer que también existen limitantes para que los profesores no aprendan, en este sentido los sistemas de creencias, como la cultura institucional juega un papel relevante.

\section{CONCLUSIONES}

El diseño del cuestionario brinda la probabilidad de identificar aquellos factores que promueven el aprendizaje en los profesores en un contexto educativo universitario. Existen estudios relacionados que marcan la necesidad de abrir la posibilidad de estructurar el conocimiento y el aprendizaje, en este sentido Lunvall's (1996) considera que la capacidad de aprender de las personas es un referente en el éxito de las organizaciones así como también en su capacidad para olvidar y aprender nuevos conocimientos para desarrollar nuevas habilidades.

En este sentido los colaboradores tienen que saber qué, saber por qué, saber cómo y saber quién. Bengt-äke \& Johnson (2006) manifiestan su preocupación en cómo se utiliza el conocimiento y el aprendizaje, expresan la necesidad de desarrollar un marco conceptual para poder dar al conocimiento y al aprendizaje los roles centrales que merecen en el análisis del cambio económico.

La economía en un concepto dinámico, implica aprender y extender la base del conocimiento ya que este elemento es un recurso económico fundamental. Según 
Torrent (2016) el docente debe estar comprometido con sus estudiantes para construir el conocimiento hacia nuevas aproximaciones del saber. Para Álvarez, Rengifo, y Cañaveral, (2017) consideran la racionalidad en la enseñanza de la economía en el sentido de que existe la posibilidad de pensar, reflexionar y actuar para recuperar otras formas de adquirir determinados saberes. Existen diversos estudios e investigaciones sobre la gestión del conocimiento en las IES, para la transferencia del conocimiento a través de una estructura o un modelo donde el uso de la tecnología juega un papel primordial, pero en la economía del aprendizaje existe la necesidad de crear un instrumento que permita diagnosticar los determinantes del aprendizaje en los profesores en un contexto educativo como primer paso orientado hacia la economía del conocimiento. Cabe señalar que una de las limitantes es la escaza literatura con respecto al tema de la economía del aprendizaje ya que algunos autores contemplan este apartado dentro del tema de la gestión del conocimiento. Este estudio tiene la posibilidad de presentar un marco de referencia para identificar los determinantes del aprendizaje que permita fomentar el progreso y la transformación social en los profesores de las IES. Es un primer paso para que los colaboradores tengan una formación y disposición para aprender y desaprender en un contexto global y emergente, para motivar e incentivar el cambio que modifique conductas hacia el logro de objetivos y el trabajo colaborativo. La presente investigación motiva para dar paso a nuevos temas de interés relacionados con la economía del aprendizaje. 


\section{REFERENCIAS}

Álvarez, A. E., Arreguit, \& Xavier. (04 de Octubre de 2019). El futuro de la universidad y la universidad del futuro. Ecosistemas de formación continúa para una sociedad de aprendizaje y enseñanza sostenible y responsable. Aula Abierta, 34. doi:https://doi.org/10.17811/rifie.48.4.2019.447-480

Álvarez, L. M., Rengifo, C. C., \& Cañaveral, L. D. (Enero de 2017). La racionalidad en la enseñanza y aprendizaje de la economía y sus implicaciones en el progreso humano. Sophia, 10. doi:http://dx.doi.org/10.18634/sophiaj.13v.1i.685

Barrios, H. K., Olivero, V. E., \& Figueroa, S. B. (Abril de 2020). Condiciones de la gestión del talento humano que favorecen el desarrollo de las capacidades dinámicas. 8. doi:http://dx.doi.org/10.4067/S0718-07642020000200055

BBVA. (2017). La competitividad de las regiones españolas ante la economía del conocimiento. España: Editorial Biblioteca Nueva.

Bengt-äke, L., \& Johnson, B. (23 de Mayo de 2006). The Learning Economy. Industry and Innovation, 21. doi:http://dx.doi.org/10.1080/13662719400000002

Calderón, M. I., Ríos, R. M., Ceccarini, \& Fernanda, M. (2008). Economía de la Educación. Argentina: Universidad Nacional de Cuyo.

Céspedes, G. S., Ceja, R. S., \& de León, C. G. (2020). Análisis de la percepción de la conducta intraemprendedora de los docentes de una IES. VinculaTégica, 15.

Céspedes, G. S., Pacheco, L. E., \& Vázquez, G. L. (2020). Análisis del modelo de competencias emprendedoras de Palacios en estudiantes de una IES. VinculaTégica, 18.

Clara, Z. M., \& Vega, Z. C. (2020). La noción de educación de calidad a nivel superior: una reflexión desde el discurso del desarrollo. Interconectando Saberes, 5(10), 10. doi:https://doi.org/10.25009/is.v0i10.2672

Cohen, L., \& Manion, M. L. (2002). Métodos de investigación educativa. México: La Muralla.

Correa, D. A., Benjumea, A. M., \& Valencia, A. A. (2019). La gestión del conocimiento: una alternativa para la solución de problemas educacionales. Revista Electrónica Educare, 27. doi:http://dx.doi.org/10.15359/ree.23-2.1

Hernández, S. R., Fernández, C. C., \& Baptista, L. P. (2014). Metodología de la investigación . México: Mc Graw Hill.

Lora, G. H., Castilla, P. S., \& Góez, F. M. (Junio de 2020). La gestión por competencias como estrategia para el mejoramiento de la eficiencia y eficacia organizacional. Saber, Ciencia y Libertad, 12. doi:https://doi.org/10.18041/2382-3240/saber.2020v15n1.6291

Lundvall, B.-Å. (Abril de 1996). The Social Dimension of The Learning Economy. DANISH RESEARCH UNIT FOR INDUSTRIAL DYNAMICS, 29.

Morduchowics, A. (2004). Discusiones de Economía de la Educación. Argentina: Losada.

ONU. (2020). Respuesta integral de las Naciones Unidas a la Covid 19: salvar vidas, proteger a las sociedades, recuperarse mejor. $O N U, 22$.

Pulgarín, F. C., Ormaza, A. J., \& Erazo, Á. J. (15 de Agosto de 2020). Gestión del capital intelectual en las organizaciones. Revista Arbitrada Interdisciplinararia KOINONIA, 26. doi:http://dx.doi.org/10.35381/r.k.v5i3.921

Quintero, R. J. (05 de Agosto de 2020). El efecto del COVID-19 en la Economía y Educación: estrategias para la educación virtual en Colombia. Revista Scientific, 12. doi:http://www.indteca.com/ojs/index.php/Revista_Scientific/oai

Ramírez, R., Espíndola, C., Ruíz, G., \& Hugueth, A. (Diciembre de 2019). Gestión del talento humano: ánalisis desde el enfoque estratégico. Información Tecnológica, 10. doi:http://dx.doi.org/10.4067/S0718-07642019000600167

Rodríguez, R. Y. (30 de Junio de 2019). Mejores prácticas para gestionar el conocimientosegún la SIO 30401. Signos, 12. doi:https://doi.org/10.15332/24631140.5090

Roncancio, R. P. (2020). La adaptación y las Instituciones de Educación Superior. Revista Iberoamericana de Educación Superior, 17. doi:http://dx.doi.org/10.4067/S0718- 
07642020000200055

Stiglitz, J., \& Greenwald, B. (2019). La creación de una sociedad del aprendizaje. Un nuevo enfoque hacia el crecimiento, el desarrollo y el progreso social. México: Paidós.

Suárez, M. A., \& Fernández, D. A. (08 de Noviembre de 2018). Economía de la educación: un análisis dialógico. Revista histórica de la educación colombiana. doi:https://doi.org/10.22267/rhec.192222.57

Supo, J. (2013). Cómo validar un instrumento. Aprende a crear y validar instrumentos como un experto. Perú: Biblioteca Nacional de Perú. Obtenido de http://www.cua.uam.mx/pdfs/coplavi/s_p/doc_ng/validacion-de-instrumentos-demedicion.pdf

Torrent, S. J. (2016). La economía del conocimiento y el conocimiento de la economía. Revista de economía, empresa y sociedad, 7.

UNESCO. (2015). www.unesco.org. Obtenido de www.unesco.org: https://es.unesco.org/node/266395 